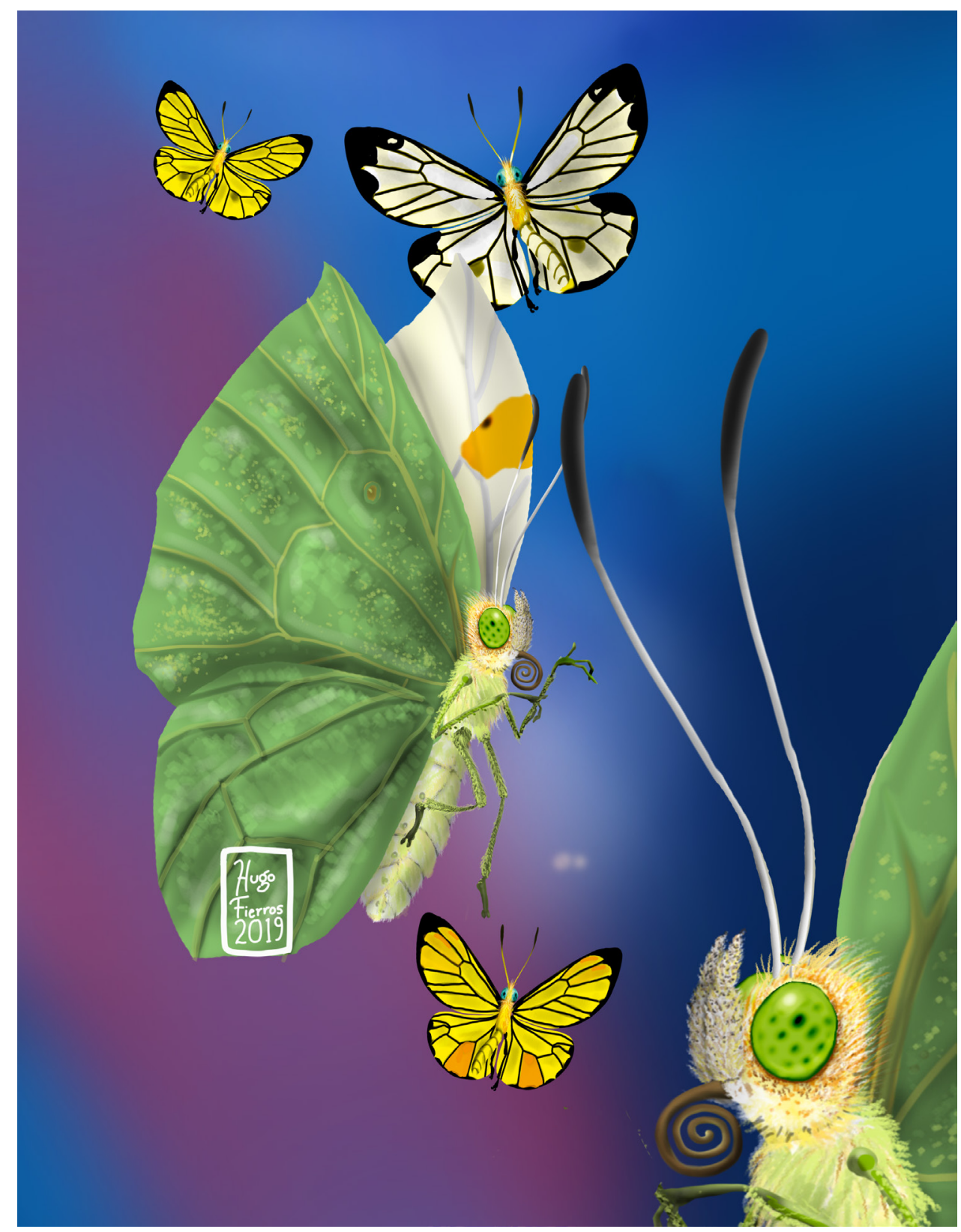

Dugesiana, Año 26, No. 2, julio 2019-diciembre 2019 (segundo semestre de 2019), es una publicación semestral, editada por la Universidad de Guadalajara, a través del Centro de Estudios en Zoología, por el Centro Universitario de Ciencias Biológicas y Agropecuarias. Camino Ramón Padilla Sánchez \# 2100, Nextipac, Zapopan, Jalisco, Tel. 37771150 ext. 33218, http://148.202.248.171/dugesiana/index.php/DUG/index, glenusmx@gmail.com. Editor responsable: José Luis Navarrete Heredia. Reserva de Derechos al Uso Exclusivo 04-2009-062310115100203, ISSN: 2007-9133, otorgados por el Instituto Nacional del Derecho de Autor. Responsable de la última actualización de este número: José Luis Navarrete Heredia, Editor y Ana Laura González-Hernández, Asistente Editorial. Fecha de la última modificación 25 de julio 2019, con un tiraje de un ejemplar.

Las opiniones expresadas por los autores no necesariamente reflejan la postura del editor de la publicación.

Queda estrictamente prohibida la reproducción total o parcial de los contenidos e imágenes de la publicación sin previa autorización de la Universidad de Guadalajara. 
Artículo

\title{
Los Mantodea en la filatelia (Insecta: Dictyoptera)
}

\author{
The Mantodea in philately (Insecta: Dictyoptera) \\ Erick Hernández-Baltazar ${ }^{1, ~ *}$ y Benigno Gómez ${ }^{2}$ \\ ${ }^{1}$ Instituto de Ciencias Biológicas, Universidad de Ciencias y Artes de Chiapas. Tuxtla Gutiérrez, Chiapas, México. \\ kcireherbal@gmail.com*; ${ }^{2}$ Departamento de Conservación de la Biodiversidad, El Colegio de la Frontera Sur. San \\ Cristóbal de Las Casas, Chiapas, México.bgomez@ecosur.mx
}

\section{RESUMEN}

Los sellos postales, desde su aparición en 1840, han abordado un sinnúmero de temáticas, incluida la biodiversidad y con ello a los insectos. El objetivo de este trabajo fue realizar un inventario de sellos postales con la representación de los Mantodea como grupo biológico. Se realizó una recopilación documental de trabajos especializados sobre entomofilatelia, revisión de grupos de Mantodea y una revisión exhaustiva en la internet. Se recopilaron 136 sellos postales en los cuales los Mantodea están representados por nueve familias, 23 géneros y 37 especies (28 válidas, una subespecie y ocho morfoespecies), lo que representa únicamente el 1.12\% de la mantidofauna mundial (2 494 especies), indicando un grado de conocimiento limitado de estas especies y escasa empatía por ellas.

Palabras clave: Mántidos, timbres postales, distribución, inventario.

\begin{abstract}
Since their appearance in 1840 postal stamps, have addressed a number of issues, including biodiversity and insects. The objective of this work was to carry out an inventory of postal stamps with the representation of the Mantodea as a biological group. A documentary compilation of specialized works on entomophilately, review of Mantodea's groups and a thorough review on the Internet. 136 postal stamps will be collected in which the Mantodea are represented by nine families, 23 genera and 37 species (28 valid species, one subspecies and eight morphospecies), which represents $1.12 \%$ of the world mantid fauna (2 494 species), indicating a limited degree of knowledge of these species and little empathy for them.
\end{abstract}

Key words: Mantids, stamps, distribution, inventory.

Filatelia es el término con el que Herpin (1864 cit pos, Palacios-Vargas 2011) definió la afición por coleccionar, clasificar sellos y documentos postales. En el caso específico de los sellos, timbres o estampillas postales, éstos son considerados como documentos o marcas que formalizan el contrato tácito entre el emisor de una carta, o cualquier otro objeto con carácter postal, y un servicio público que asume la responsabilidad de transportar y entregar dicha carta u objeto (Ferreira 2003). Los sellos postales, desde su aparición en 1840, han abordado un sinnúmero de temáticas, incluida la biodiversidad (Gómez y Junghans 2016).

Dentro de la biodiversidad abordada como tema postal, los artrópodos son uno de los grupos bien representados. Dentro de los Arthropoda, los Hexapoda son los más diversos a nivel mundial con más de un millón de especies registradas (Giribert y Edgecombe 2013, Gullan y Cranston 2014). Es así como, por el número de especies, su abundancia y las interacciones que pueden establecer con la humanidad, así como con su entorno, los insectos tienen un éxito notorio y no podían dejar de ser empleados en el arte de la filatelia (Costa-Neto 2002, Gómez y Junghans 2002, Palacios-Vargas y Navarrete-Heredia 2003, Pollock 2000). En este papel desempeñan un valor económico y cultural, ya que las estampas se compran para uso práctico, por valor estético o belleza (Leather 2015). A esta representación insecto-estampa se le conoce como entomofilatelia (Hamel 1990).

Dentro de los insectos se encuentran los Mantodea, un grupo perteneciente al superorden Dictyoptera que comprenden 2494 especies válidas a nivel mundial (Wieland y Svenson 2018). Se distribuyen en hábitats tropicales y subtropicales del mundo, desde las selvas hasta los desiertos (Patel et al. 2016, Wieland y Schütte 2017). Este grupo, a pesar de ser considerados raros (por su baja riqueza de especies y ser crípticos en los ambientes donde habitan), no han pasado desapercibidas para su representación en la filatelia de diversas partes del mundo (Ehrmann 2002, Roy y Ehrmann 2009).

\section{MATERIAL Y MÉTODOS}

Se realizó una recopilación documental de trabajos que hicieran mención gráfica de Mantodea en sellos postales alrededor del mundo, documentos especializados como Costa-Neto (2002), Domingo (1997), Hamel (1990), Mendoza et al. (2006), Palacios-Vargas (2011) y PalaciosVargas y Navarrete-Heredia (2003), así como páginas electrónicas en internet dedicadas a la entomofilatelia (Entomophila 2019, Insects on Stamps 2019, Krüger 2019, Maes 2019, StampWorld 2019, The Philately 2019, Wopa 
2019). Aunado a esto, los trabajos de Ehrmann (2002), Roy (2004) y Roy y Ehrmann (2009) sobre los Mantodea del mundo y revisión de géneros (Choeradodis y Zoolea) respectivamente, son un recurso de información particular para aclarar la representación de este grupo en la filatelia. Los resultados se agruparon en una base de datos de Microsoft Access $($, siendo los siguientes campos de captura: 1) No. Identificación, 2) País, 3) Año, 4) Superfamilia, 5) Familia, 6) Género y 7) Especie válida. El arreglo taxonómico sigue la propuesta de Schwarz y Roy (2019).

\section{RESULTADOS}

Se recopilaron 136 sellos postales con imágenes de Mantodea, los cuales representan a nueve familias, 23 géneros, 28 especies válidas, una subespecie y ocho morfoespecies (Figura 1). La entomofilatelia de este grupo se distribuye en 72 países (Figura 2). A continuación, se presenta el compendio del grupo Mantodea representado en la filatelia mundial (el asterisco * indica una morfoespecie o especie inválida y $s / a$ sin año):

\section{Acanthopidae}

*Acontista sp.: Lesoto (2002)

\section{Coptopterygidae}

Coptopteryx gayi Blanchard, 1851: Chile (1948, 1949)

\section{Deroplatyidae}

Deroplatys desiccata Westwood, 1839: Somalia (2002), Malasia (2013), África Central (2013)

\section{Empusidae}

Blepharopsis mendica (Fabricius, 1775): España (1965), Emiratos Árabes Unidos (1998), Tanzania (2013)

Empusa fasciata Brulle, 1832: Turquía (1981)

*Empusa sp.: África Central (1997), República Democrática del Congo (2010)

Empusa pennata (Thunberg, 1815): San Vicente y las Granadinas (2005), Chad (2014)

Hemiempusa capensis Burmeister, 1838: Namibia (1987)

\section{Eremiaphilidae}

Iris oratoria (Linne, 1758): Chipre del Norte (2013)

\section{Galinthiadidae}

Harpagomantis tricolor Linne, 1758: Gambia (2003)

\section{Hymenopodidae}

Creobroter gemmatus Saussure, 1869: China (2012)

*Creobroter sp.: Islas Turcas y Caicos (2002)

Hymenopus coronatus Olivier, 1792: Malasia $(1998,2014)$, Somalia (2002), Bélgica (2015), Guinea-Bisáu (2015), Islas Salomón (2015)

*Pseudocreobotra sp.: Islas Turcas y Caicos (2002)

Pseudocreobotra wahlbergi Stål, 1871: República de Rodesia (1969), Zimbabue (1988), Países Bajos (1993),
Níger (2000), Ghana (2002), Santo Tomé y Príncipe (2007), República Centroafricana (2015)

\section{Mantidae}

Choeradodis strumaria (Linne, 1758): Belice (1995), Guinea-Bisáu (2010)

*Choeradodis sp.: Mozambique (2013)

Hierodula venosa Olivier, 1792: Indonesia (2003), Vietnam (2009)

Hierodula patellifera Serville, 1839: Bután (1969)

Mantis religiosa Linne, 1758: Estados Unidos (1950, 1975, 1980), Costa de Marfil (1979, 1980), Alto Volta (1981), Escocia (1982), Isla de Navidad (1987), Camboya (1988), Dominica (1988), Corea del Norte (1990), Suiza (1991), Bulgaria (1992), Mali (1994), República Checa (1995, 2007), Bután (1997), Moldavia (1997, 2016), Senegal (1997), Qatar (1998), Pakistán (1999), Sudáfrica $(2000,2005)$, Chipre del Norte (2000), Bolivia (2001), Liberia (2001), Madagascar (1999, 2001, 2016), Somalia (2002), Australia (2003), Tayikistán (2003), Kirguistán (2004), Mongolia (2004), San Vicente y las Granadinas (2005), Malta (2005), Austria (2006, 2012, 2015), San Tomé y Príncipe (2007), Guinea (2007), Vietnam (2009), República de Altái (2010), Tanzania (2010), Benín (2011), República del Congo (2011), Mozambique (2014), Ucrania (2018)

*Polyspilota sp.: Uganda (2002)

Polyspilota seychelliana Giglio-Tos, 1911: Seychelles (1988)

Rhombodera basalis de Haan, 1842: República Centroafricana (2011)

Sphodromantis gastrica Stål, 1858: Lesoto (1978), Botsuana (1981)

Sphodromantis lineola Burmeister, 1838: Alto Volta (1966), Níger (1987)

Sphodromantis viridis Forskal, 1775: Mali (1977), Ruanda (2002), Senegal (2010), Sudáfrica (2010)

*Sphodromantis sp.: Malaui (1970), Benín (2002)

Stagmatoptera septentrionalis Saussure \& Zehntner, 1894:

San Vicente y las Granadinas (Isla Unión) (2011)

Stagmomantis carolina (Johansson, 1763): San Vicente y las Granadinas $(2001,2002)$

Stagmomantis limbata Hahn, 1835: San Vicente y las Granadinas (1979)

* Stagmomantis sp.: Costa Rica (2007)

Tenodera aridifolia Stoll, 1813: China (1992), Japón (1995), Vietnam (2009)

*Tenodera aridifolia sinensis: Granada (2002)

Tenodera sinensis Saussure, 1871: El Salvador (1970), China $(s / a)$

Tisma freyi Brancsik, 1892: Madagascar (1966)

Zoolea minor Giglio-Tos, 1914: Brasil (1987)

\section{Miomantidae}

Miomantis paykullii Stål, 1871: Portugal (2011) 


\section{DISCUSIÓN}

Esta diversidad de timbres postales representa únicamente el $1.12 \%$ de la mantidofauna mundial (2 494 especies; Wieland y Svenson 2018), un pequeño porcentaje que no es raro en este grupo, considerado la baja riqueza registrada en comparación con otros Hexapoda.

Taxonómicamente la familia mejor representada fue Mantidae con 11 géneros y 16 especies válidas, una subespecie y cuatro morfoespecies, pero esto no tiene nada de extraño, al ser esta la familia más diversa en el orden Mantodea, con poco menos del 50\% de la diversidad mundial (1 016 especies; Wieland y Svenson 2018). La especie más frecuentemente representada es Mantis religiosa con 49 apariciones, desde 1950 (Estados Unidos) hasta 2018 (Ucrania), siendo esta una especie de distribución casi mundial, desde América del Norte hasta Asia, África y Europa, sin embargo, está ausente en América Tropical y Oceanía (Moulin 2018). En contraste, son 13 especies de Mantodea que están representados solo una vez en la historia de la filatelia, indicando un grado de conocimiento muy escueto de estas especies y escasa empatía por parte de los países.

Se recopilaron ocho sellos de morfoespecies, siendo los géneros Acontista, Creobroter, Choeradodis, Empusa, Polyspilota, Pseudocreobotra, Sphodromantis y Stagmomantis representados en países de África Austral, Central, Occidental y Oriental, las Bahamas (Islas Turcas y Caicos) y América Central (Costa Rica). Lo anterior debido a la escasez de información visual para su identificación, señalando la falta de caracteres morfológicos visibles en los timbres como principal impedimento para llegar a nivel específico, no obstante, se puede intuir la identidad de algunos ejemplares, tal es el caso del género Polyspilota Burmeister, 1838 que potencialmente correspondería a Polyspilota aeruginosa (Goeze, 1778) o Polyspilota saussurei Bormans, 1881, con distribución en Tanzania (Patel y Singh 2016), muy cercano a Uganda, cuyo país emitió el sello. El caso del género Sphodromantis Stål, 1871, que podría ser Sphodromantis citernii kenyana La Greca \& Lombardo, 1987, que presenta distribución en Malaui (Patel y Singh 2016), país donde se emite el sello. Un caso particular es el género Acontista, cuya distribución ocurre exclusivamente en la región neotropical (Rivera 2010), sin embargo, Lesoto en el 2002, emitió un sello alusivo a este género, lo que demuestra que la filatelia de un Mantodea no siempre indica que esa especie tenga distribución en el país emisor (Gómez et al. 2015).

Diversos países del mundo han presentado interés para inmiscuir en sus timbres postales imágenes de Mantodea desde 1948, siendo el más frecuente San Vicente y las Granadinas con seis emisiones. En 1948 fue Chile el primer país en representar a Coptopteryx gayi en un timbre postal, y Ucrania en el 2018 es el último país que hasta el momento con $M$. religiosa. Durante el periodo de revisión de la emisión de sellos postales (1948-2018), existen algunos con motivos de conmemoración a entomólogos importantes, que en sus estudios describieron nuevas especies o realizaron trabajos descriptivos de varios grupos de los Hexapoda y en cuyos timbres aparecen ilustrados los Mantodea. Estos son los casos, por citar alguno, como el de Jean-Henri Fabre, Félix Édouard Guérin-Méneville y John Obadiah Westwood en los países de Chad, Francia y República Centroafricana (Figura 3). Aunado a lo anterior, en Pakistán (1999) se dedicó una estampilla al Dr. Muhammad Afzal Hussain Qadri, entomólogo que realizó estudios particularmente en el mecanismo de perforación y succión de insectos, así como en el campo de la genitalia de Orthoptera (Figura 3). Por otra parte, los sellos que ilustran motivos de aniversario, tales como el emitido por Mozambique en 2014 que representó el $150^{\circ}$ Aniversario de la novela de Julio Verne ("Viaje al Centro de la Tierra"), de 1864 con un ejemplar de Mantodea, potencialmente M. religiosa (Figura 3) o el sello de Brasil por el $50^{\circ}$ Aniversario de la Sociedad Brasileña de Entomología (Roy y Ehrmann 2009), que erróneamente nombraron en un sello postal a Zoolea minor como "Zoolea lopiceps" (Costa-Neto 2002, Roy y Ehrmann 2009). Esto último, no es el único caso en el que las estampillas emitidas presentan errores de nomenclatura, tal es el caso de "Creobroter gemmata" emitido en China (2012), cuyo nombre válido es Creobroter gemmatus y la subespecie "Tenodera aridifolia sinensis" de Granada (2002), posiblemente su verdadera identidad se vea más reflejada en las subespecies Tenodera aridifolia aridifolia Stoll, 1813 y Tenodera aridifolia brevicollis Beier, 1933 por la distribución más afín al continente asiático (Patel y Singh 2016). En otros casos, simplemente han recibido modificaciones nomenclaturales, como el timbre postal de China emitido en 1992 con el nombre Paratenodera aridifolia y de acuerdo con Otte et al. (2019) el nombre válido es Tenodera aridifolia o el caso del sello emitido por Indonesia en 2003 de Hierodula vitrea, la cual es actualmente sinonimia de $H$. venosa (Otte et al. 2019).

Finalmente, es de suma importancia que la comunidad entomológica (estudiantes, profesionistas y aficionados) contribuya activa y entusiastamente en la emisión de sellos postales con el tema de los artrópodos, promoviendo el interés de servicios postales a nivel mundial para plasmar en sus estampas el valor cultural y biológico. Como ya mencionaron algunos autores (Child 2008, Scott 1995), los sellos postales son considerados una fuente semiótica, con la capacidad impresionante de transmitir varios mensajes en un espacio muy reducido, los cuales se pueden aprovechar para hacer la difusión de la bioecología, taxonomía y diversidad de los Mantodea, así como hacer conciencia de su conservación.

\section{LITERATURA CITADA}

Child, J. (Ed). 2008. Miniature messages: The semiotics and politics of Latin American postage stamps. Duke University Press Books, Durham, North Carolina.

Costa-Neto, E.M. 2002. Etnoentomofilatelia: os insetos na arte filatelico. Bioikos, 16(1/2): 61-67. 
Domingo, J. (Ed). 1997. Catálogo de sellos temáticos fauna, mariposas y otros insectos. Ediciones Domfil, Sabadell, España.

Ehrmann, R. (Ed). 2002. Mantodea. Gottesanbeterinnen der Welt. Natur und Tier Verlag $\mathrm{GmbH}$, Münster, Deutschland.

Entomophila. 2019. The stamps about invertebrates. http:// entomophila.chez.com/. Fecha de consulta: 20 de enero de 2019.

Ferreira, L.E. (Ed). 2003. A certain look at philately. Edições Húmus, Lda., Clube Nacional de Filatelia, Portugal.

Giribert, G. and G.D. Edgecombe. 2013. The Arthropoda: A phylogenetic framework. (pp. 17-40). In: Minelli, A., G. Boxshall and G. Fusco (Eds.). Arthropod Biology and Evolution. Berlin, Germany.

Gómez, B. y C. Junghans. 2002. Los Scarabaeoidea (Insecta: Coleoptera) en la filatelia. Cuadernos de Biodiversidad, (11): 10-14.

Gómez, B. y C. Junghans. 2016. Los alacranes (Arachnida: Scorpionida) en la filatelia. Dugesiana, 23(1): 37-43.

Gómez, B., M.R. Domínguez y C. Junghans. 2015. Las cucarachas (Blattodea) representadas en los sellos postales (entomofilatelia). (pp. 77-82). En: CastañoMeneses, G. y J.L. Navarrete-Heredia (Coords.). Avances en la Entomología Cultural Mexicana. Jalisco, México.

Gullan, P. J. and P. S. Cranston. (Eds). 2014. The insects: an outline of entomology. Wiley-Blackwell, Oxford, England.

Hamel, D.R. 1990. Insects on stamps. American Entomologist, 36(4): 273-282.

Insects on Stamps. 2019. http://www.asahi-net. or.jp/ CH2M-NITU/indexe.htm. Fecha de consulta: 25 de enero de 2019.

Krüger, B. 2019. BK Makro. http://www.bkmakro.de/ Makro/frmantibriefm.htm. Fecha de consulta: 23 de febrero de 2019.

Leather, S.R. 2015. Influential entomology: a short review of the scientific, societal, economic and educational services provided by entomology. Ecological Entomology, 40: 36-44.

Maes, J.-M. 2019. EntomoFilatelia- Sellos postales del mundo sobre insectos. http://bio-nica.info/ entomofilatelia/index.html. Fecha de consulta: 28 de enero de 2019.

Mendoza, M., I. Pacheco, M.A. Sarmiento y M.L. Zurita. 2006. Entomofilatelia en México: un tributo a la amistad de los insectos. Boletín de la Sociedad Entomológica Aragonesa, 38: 443-449.

Moulin, N. 2018. Liste commentée et catalogue illustré des Mantodea du Gabon. Les cahiers de la fondation Biotope, (24): 2-60.

Otte, D., L. Spearman and M. Stiewe. 2019. Mantodea Species File Online. Versión 5.0/5.0. http://mantodea. speciesfile.org/. Fecha de consulta: 27 de febrero de
2019.

Palacios-Vargas, J.G. 2011. Entomofilatelia: Las estampillas postales con insectos y otros artrópodos en el mundo. (pp. 15-16). En: Navarrete-Heredia J.L., G. Castaño-Meneses y G.A. Quiroz-Rocha (Eds.). Facetas de la Ciencia: Ensayos sobre Entomología Cultural. Universidad de Guadalajara, Jalisco.

Palacios-Vargas, J.G. y J.L. Navarrete-Heredia. 2003. Entomofilatelia, un aspecto de la entomología cultural. (pp. 107-115). En: Llorente-Bousquets, J. y J.J. Morrone (Eds.). Biodiversidad, Taxonomía y Biogeografia de Artrópodos de México: Hacia una síntesis de su conocimiento, volumen III. Facultad de Ciencias, UNAM, Ciudad de México.

Patel, S. and R. Singh. 2016. Update checklist and distribution of Mantidae (Mantodea: Insecta) of the World. International Journal of Research Studies in Zoology, 2(4): 17-54.

Patel, S., G. Singh and R. Singh. 2016. A checklist of global distribution of Liturgusidae and Thespidae (Mantodea: Dictyoptera). Journal of Entomology and Zoology Studies, 4(6): 793-803.

Pollock, D.A. 2000. Insects on stamps- good guys and bad guys. Newsletter, The Entomological Society of Manitoba, 27(2): 1-14.

Rivera, J. 2010. A historical review of praying mantid taxonomy and systematics in the Neotropical region: state of knowledge and recent advances (Insecta: Mantodea). Zootaxa, 2638: 44-64.

Roy, R. 2004. Révision et phylogénie des Choeradodini Kirby, 1904 (Dictyoptera, Mantidae). Bulletin de la Société entomologique de France, 109(2): 113-128.

Roy, R. et R. Ehrmann. 2009. Révision du genre Zoolea Audinet-Serville [Mantodea, Mantidae, Vatinae]. Revue française d'Entomologie (N.S.), 31(1): 1-22.

Schwarz, C.J. and R. Roy. 2019. The systematics of Mantodea revisited: an updated classification incorporating multiple data sources (Insecta: Dictyoptera). Annales de la Société entomologique de France (N.S.), 55(2): 101-196.

Scott, D. (Ed). 1995. European stamp design: a semiotic approach to designing messages. Academy Editions, London, England.

Shorthouse, D.P. 2010. SimpleMappr, an online tool to produce publication-quality point maps. http://www. simplemappr.net. Fecha de consulta: 25 de enero de 2019.

StampWorld. 2019. El catálogo de sellos más completo del mundo. http://www.stampworld.com/es/. Fecha de consulta: 15 de febrero de 2019.

The Philately. 2019. Insects. https://thephilately.com/topic/ insects. Fecha de consulta: 25 de marzo de 2019.

Wieland, F. und K. Schütte. 2017. Fortschritte und Perspektiven in der Erforschung der Evolution und Phylogenie der Mantodea (Insecta: Dictyoptera). Entomologie heute, 29: 1-23. 
Wieland, F. and G.J. Svenson. 2018. Biodiversity of Mantodea. (pp. 389-416). In: Foottit, R.G. and P.H. Adler (Eds.). Insect Biodiversity: Science and Society, Volume II. New Jersey, United States.

Recibido: 20 de mayo 2019

Aceptado: 20 de junio 2019
Wopa + . 2019. Stamps and coins. https://www.wopastamps.com/. Fecha de consulta: 12 de enero de 2019.

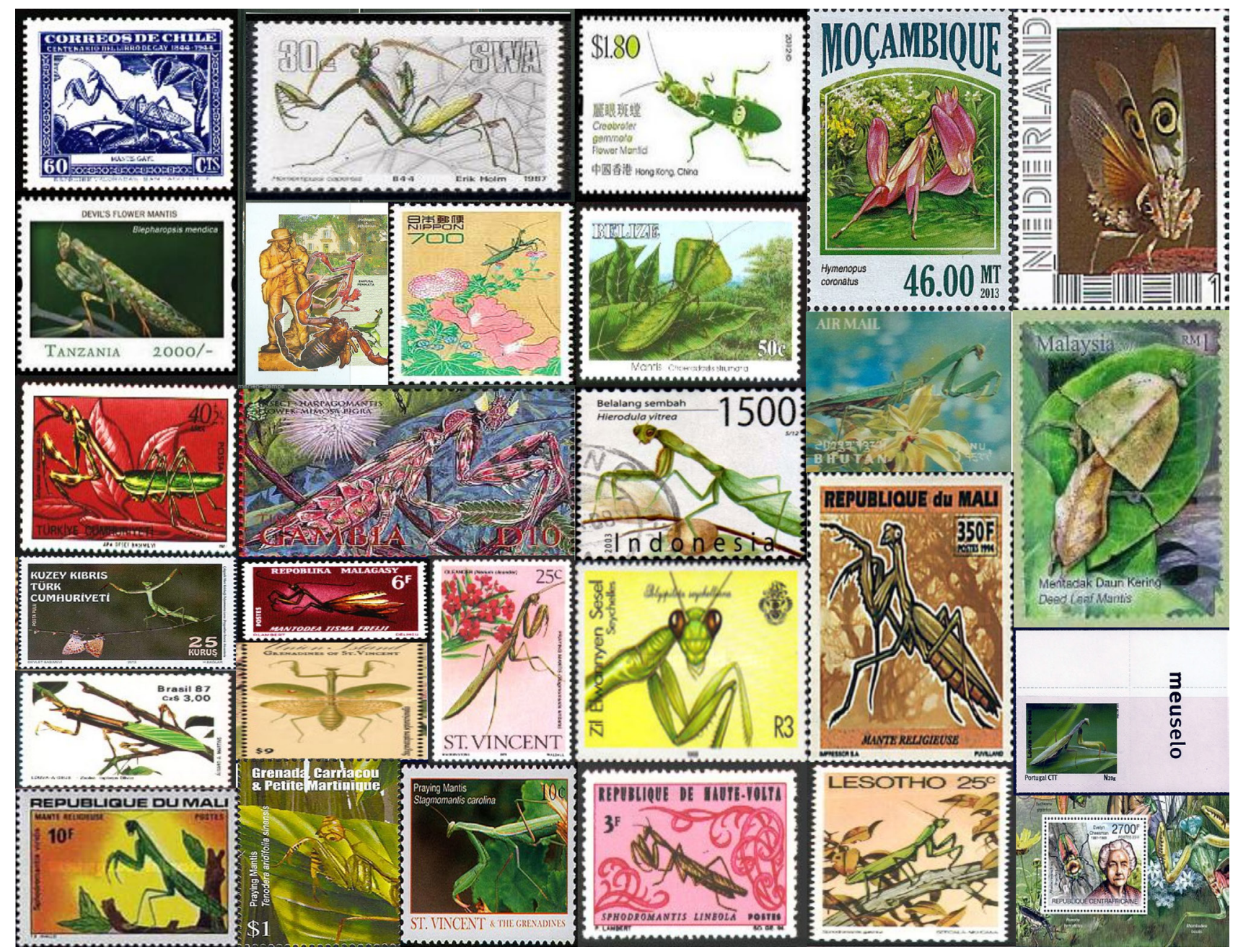

Figura 1. Compendio de las especies válidas de Mantodea representadas en la filatelia mundial. 


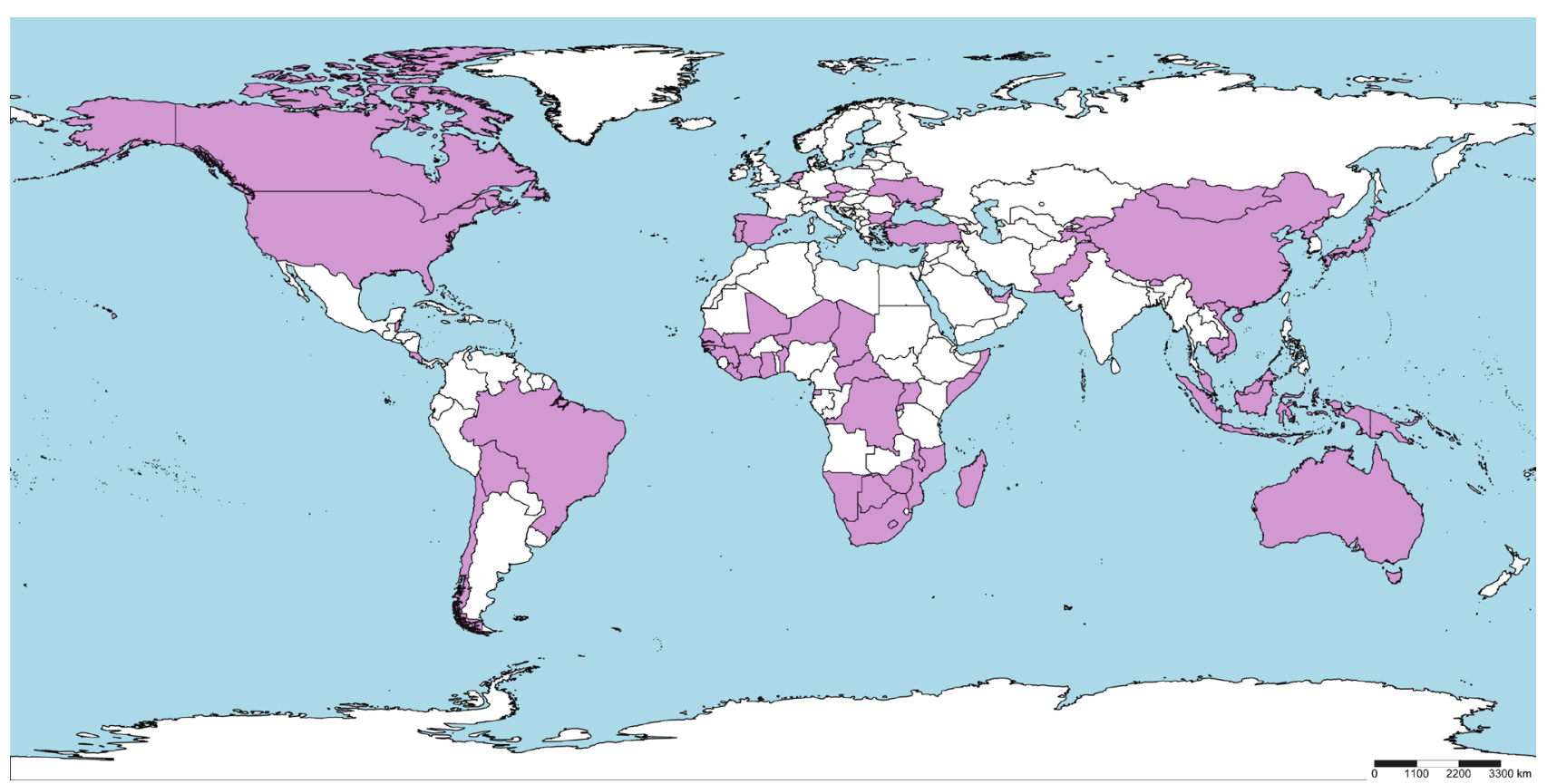

Figura 2. Mapa de distribución de los Mantodea en la filatelia (Fuente: Shorthouse, 2010).

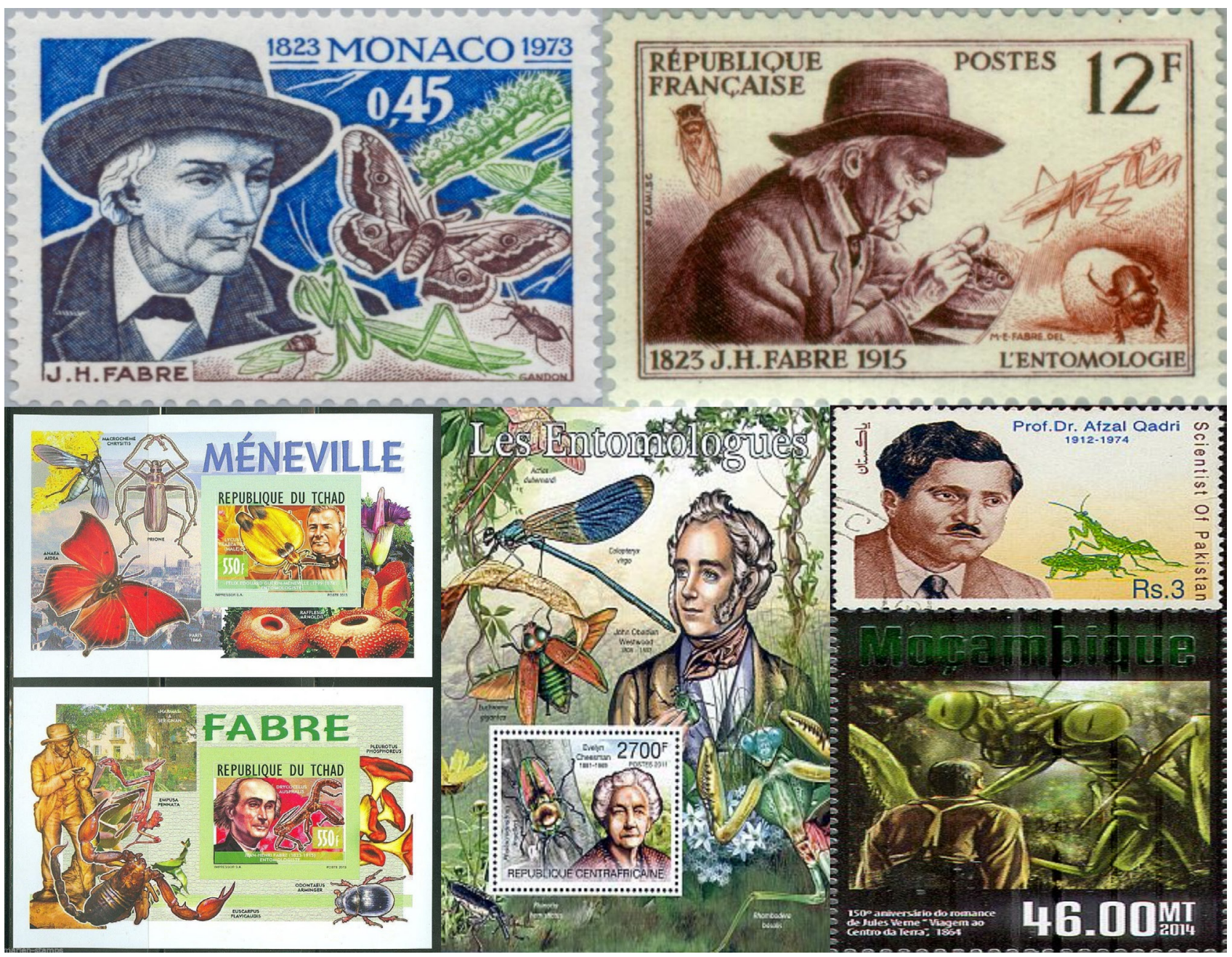

Figura 3. Compendio de los entomólogos en la filatelia, con alusión a los Mantodea. 